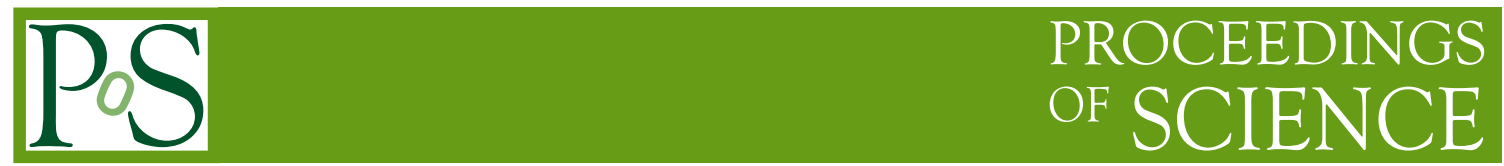

\title{
Luminosity measurement at muon collider
}

\author{
C. Giraldin, ${ }^{a, *}$ L. Buonincontri, ${ }^{b, a, *}$ U. Dosselli, ${ }^{b}$ D. Lucchesi ${ }^{a, b}$ and L. Sestini ${ }^{b}$ \\ ${ }^{a}$ University of Padova, Via Marzolo 8, 35131 Padova, Italy \\ ${ }^{b}$ Istituto Nazionale di Fisica Nucleare Sezione di Padova, Via Marzolo 8, 35131 Padova, Italy \\ E-mail: carlo.giraldin@studenti.unipd.it, laura.buonincontri@pd.infn.it, \\ umberto.dosselli@pd.infn.it, donatella.lucchesi@pd.infn.it, \\ lorenzo.sestini@pd.infn.it
}

The precise determination of the luminosity in a collider is of crucial importance for any physics cross sections measurement since it directly translates to the precision of the cross section determination. In a muon collider high intensity beams are necessary to achieve the target luminosity, and due to the muon decay they generate very high fluxes of particles that arrive to the detector. Ad-hoc shielding structure has been designed to mitigate the effect of the beam-induced background on the detector located in the backward and forward regions. Therefore, the instrumentation for the measurement of the luminosity can not be placed, as in the LHC experiments, in these regions. In this paper an alternative way to determine such a fundamental parameter is proposed, taking inspiration from flavour factories such as Belle2 and BESIII, where the luminosity is measured by counting $e^{+} e^{-} \rightarrow e^{+} e^{-}$Bhabha events, whose cross section is theoretically known with high precision. At muon collider the large angle muon Bhabha $\left(\mu^{+} \mu^{-} \rightarrow \mu^{+} \mu^{-}\right)$events are used, and here the first results on the precision obtained at $1.5 \mathrm{TeV}$ center of mass energy by using full detector simulation, taking into account the beam-induced background effects, are presented.

The Ninth Annual Conference on Large Hadron Collider Physics - LHCP2021

7-12 June 2021

Online

${ }^{*}$ Speaker 


\section{Introduction}

The instantaneous luminosity is one of the most important parameters that characterizes the performances of a collider. It can be directly determined through the measurement of the parameters of the colliding beams:

$$
\mathcal{L}=n_{b} \cdot \frac{N_{1} N_{2} \cdot f}{A_{\text {eff }}}
$$

where $n_{b}$ and $f$ are known since they are parameters of the machine and are respectively the number of bunch and the revolution frequency in the collider. $N_{1,2}$, the average number of particles per bunch, and $A_{\text {eff } f}$, the effective area, can be determined by dedicated detectors, such as luminometers, that are installed in the forward region of the detector and are used in combination to Van der Meer scan method [1] . A different approach is used in $e^{+} e^{-}$experiments like Belle2[2] and BESIII[3], where the integrated luminosity $L$ is calculated by counting the number of events $N_{e v}$ of a process whose cross section $\sigma$ is theoretically know with high precision as:

$$
L=\frac{N_{e v}}{\epsilon \cdot \sigma}
$$

where $\epsilon$ is the reconstruction efficiency. The most used process is the Bhabha scattering $\left(e^{+} e^{-} \rightarrow\right.$ $e^{+} e^{-}$) where, for example, the theoretical uncertainty on $\sigma$ at $\sqrt{s}=1-10 \mathrm{GeV}$ is $0.1 \%$ at large angle [4]. At the muon collider, this second method is pursued, and this paper presents the results obtained on the estimation of the precision on $L$ that can be achieved at $\sqrt{s}=1.5 \mathrm{TeV}$. The contribution of the theoretical calculation to the final luminosity uncertainty is not considered.

\section{Muon collider and beam-induced background}

The muon collider is one of the proposed future particle accelerator projects where opposite sign muon beams are made collide at multi-TeV center of mass energies [5]. Such high center of mass energies can be reached since muons are elementary particles, then all the beam energy is available for the interaction. Moreover the energy loss due to synchrotron radiation and beamstrahlung is negligible for muons at such energies. To reach the luminosity target scheduled for this machine $\left(2 \cdot 10^{34} \mathrm{~cm}^{-2} \mathrm{~s}^{-1}\right.$ at $\sqrt{s}=3 \mathrm{TeV}$ and $4 \cdot 10^{35} \mathrm{~cm}^{-2} \mathrm{~s}^{-1}$ at $\left.\sqrt{s}=10 \mathrm{TeV}\right)$, beams of the order of $2 \cdot 10^{12}$ muons are necessary. The beam-induced background (BIB) at the muon collider is generated by the decay of muons in such dense circulating beams. Electrons/positrons and neutrinos produced by the muons decay can interact with the material of the surrounding machine and the machine-detector interface (MDI) components and produce a high flux of secondary and tertiary particles that can reach the detector and affect its performances. Detailed simulations of the machine optics, lattice elements and design of the interaction region and the MDI are essential to characterize the amount and the composition of the BIB particles at the detector. The BIB characteristics at a muon collider have been studied for $\sqrt{s}=1.5 \mathrm{TeV}[6]$ on the basis of the machine design developed by the Muon Accelerator Program (MAP) [7] collaboration. In figure 1 left a scheme of the beam-pipe, the MDI and the muon collider detector is shown. Two cone-shaped tungsten nozzles [8] (green cones in figure 1 left), with an angular aperture of $\sim 10^{\circ}$ for $\sqrt{s}=1.5 \mathrm{TeV}$, are inserted in the detector to reduce the flux of particles of order of magnitudes. Despite of the presence of the nozzles, a large number of BIB particles $\left(\sim 10^{10}\right)$ survive and reach the detector, mainly low energy photons and 

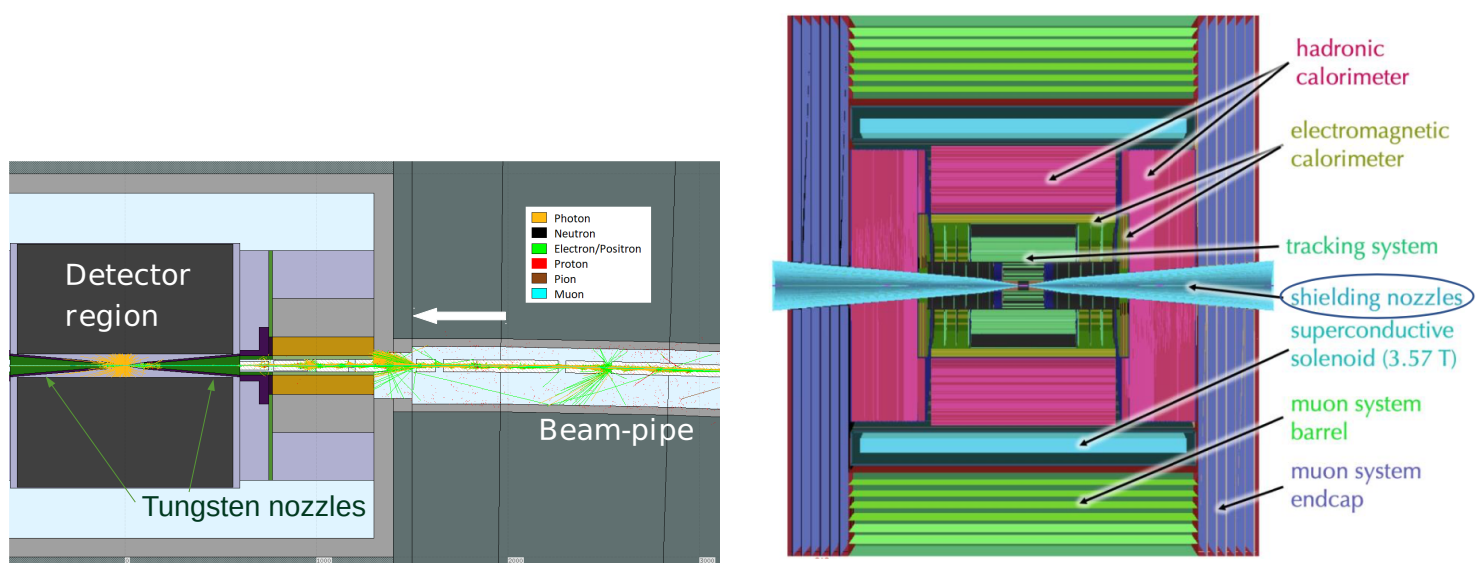

Figure 1: Left: schematic view of the machine-detector interface at the muon collider and the muon detector (dark grey)[6]. BIB particles are listed in the legend. Neutrons are not included in the picture. Right: section of the detector in the longitudinal plane with respect to the beam pipe.
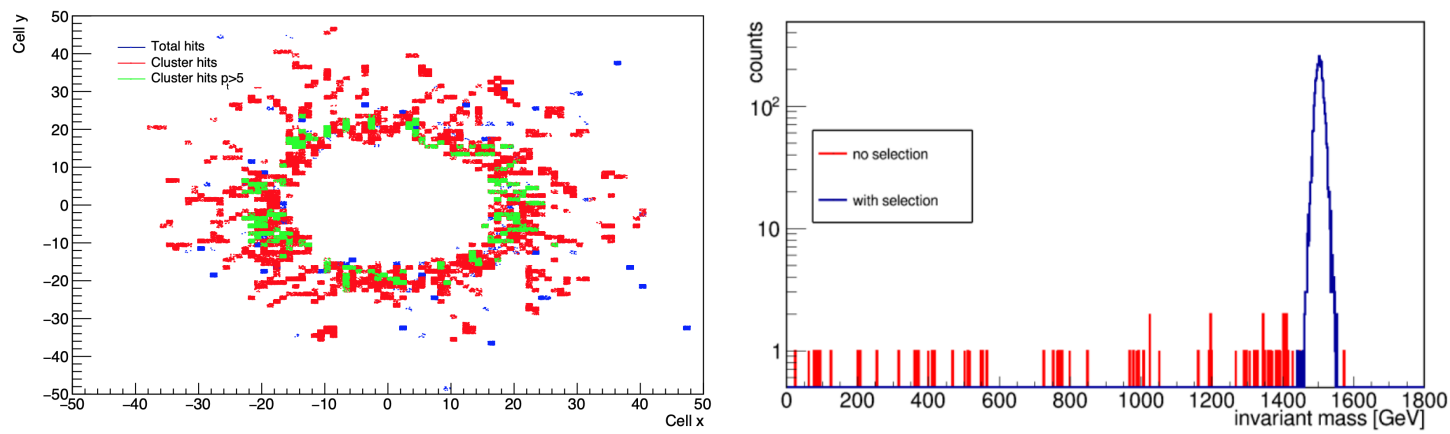

Figure 2: Left: BIB hits spatial distribution in the muon system endcap. In blue: hits not merged to form cluster. In red: hits merged to form clusters. In green: hits that are merged to form clusters associated to muons with $P_{T}>5 \mathrm{GeV}$. Right: Invariant mass distribution of the muons in the final state of the $\mu^{+} \mu^{-} \rightarrow \mu^{+} \mu^{-}$process. In red before the application of selection cuts, in blue after the selection cuts.

electrons/positrons, neutrons and charged hadrons. Sensors providing information about the energy released by particles, the time of arrival with respect the bunch crossing and the position in the detector can be employed to further reduce the amount of BIB particles. The $\sqrt{s}=1.5 \mathrm{TeV}$ BIB effects on the muon detector have been evaluated by looking at its distribution on the muon system. The schematic view of the longitudinal section with respect to the beam axis of the detector [9] [10] used for the full simulation is shown in figure 1 right. In cyan are represented the tungsten nozzles, optimized for $\sqrt{s}=1.5 \mathrm{TeV}$. The muon detectors cover the outermost region and is composed by seven barrel (in green in figure1 right) and six endcap layers (in blue in figure 1 right) of resistive plate chambers (RPC) with a cell size of $30 \times 30 \mathrm{~mm}^{2}$, interleaved in the magnet's iron return yoke. Just a small percentage $(\sim 8 \%)$ of BIB primary particles reach the muon system. Figure 2 left shows the hits spatial distribution in the muon system endcap generated by BIB particles. BIB hits in the muon system are concentrated around a region small with respect to the whole layer region of $500 \times 500$ cells. The white region at the center of the picture is the hole in the endcap that hosts the tungsten nozzle and the beam-pipe. In the barrel no BIB clusters are reconstructed. 


\section{Simulated data analysis and Results}

The presence of the nozzle prevents the installation of luminometers in the forward region of the muon collider detector and the measurement of the luminosity with the Van der Meer's scan method. For this reason the indirect method for the luminosity measurement based on the counting of Bhabha $\mu^{+} \mu^{-} \rightarrow \mu^{+} \mu^{-}$events is applied. Muons with emission angle $\theta$ respect to the beam-pipe $30^{\circ}<\theta<150^{\circ}$ are selected to study the $\mu$-Bhabha process, since the BIB effects in this angular region are negligible. The characteristics of the $\mu$-Bhabha process in the selected angular region, have been studied through the analysis of a simulated sample of $100000 \mu$-Bhabha events at $\sqrt{s}=1.5 \mathrm{TeV}$, generated with Phytia[11] Monte Carlo and reconstructed by using the Particle Flow algorithm[12] that exploits both the information from the tracking system and the clusters, released the muon detector. Two opposite sign muons (having at least one cluster in the muon detector) are selected, both with transverse momentum $P_{t}>130 \mathrm{GeV}$. The angle between muons direction pair is required to be $\Delta \theta>3.08 \mathrm{rad}$ and the di-muons invariant mass $1440<M_{\mu \mu}<1560 \mathrm{GeV}$. Applying these cuts, the reconstruction efficiency $\epsilon$ of the $\mu$-Bhabha process in the selected angular region is found to be around $95 \%$. The selection cuts, obtained from the previous analysis, were applied to a sample of $100000 \mu^{+} \mu^{-} \rightarrow \mu^{+} \mu^{-}$events at $\sqrt{s}=1.5 \mathrm{TeV}$, generated with Pythia Monte Carlo, including all the possible processes with at least two muons in the final state. In figure 2 right invariant mass of the $\mu^{+} \mu^{-}$final state pair is shown before (in red) and after (in blue) the selection cuts. The low invariant mass tail due to muons from the physics background is removed by the selection cuts. The number of selected $\mu$-Bhabha events after the cuts is scaled to obtain the number of $\mu$-Bhabha events expected in one year of data taking $\left(10^{7} \mathrm{~s}\right)$. Assuming an instantaneous luminosity $\mathcal{L}=1.25 \times 10^{34} \mathrm{~cm}^{-2} \mathrm{~s}^{-1}$ it is found to be $N_{\text {Bhabha }}=198762$. Neglecting the uncertainty about reconstruction efficiency, the uncertainty on the integrated luminosity can be calculated as:

$$
\frac{\Delta L_{i n t}}{L_{i n t}}=\sqrt{\frac{\Delta N_{e v}{ }^{2}}{N_{e v}{ }^{2}}+\frac{\Delta \sigma_{B}{ }^{2}}{\sigma_{B}{ }^{2}}}=\left(\frac{\Delta N_{e v}}{N_{e v}}\right) \oplus\left(\frac{\Delta \sigma_{B}}{\sigma_{B}}\right)
$$

the first term refers to statistical uncertainty, and the second refers to the theoretical uncertainty on the cross section of the Bhabha process. The statistical uncertainty on integrated luminosity obtained with this analysis results to be:

$$
\frac{\Delta N_{\text {Bhabha }}}{N_{\text {Bhabha }}}=\frac{1}{\sqrt{N_{\text {Bhabha }}}}=0.002
$$

\section{Conclusions and Future Prospects}

From this preliminary study on the measurement of the luminosity at the muon collider, the statistical uncertainty obtained on the integrated luminosity at $\sqrt{s}=1.5 \mathrm{TeV}$ center of mass energy, assuming an instantaneous luminosity $\mathcal{L}=1.25 \times 10^{34} \mathrm{~cm}^{-2} \mathrm{~s}^{-1}$ and one year of data taking, is found to be around $0.2 \%$. However to complete this study it is necessary to have the estimate on the precision of the cross section of $\mu$-Bhabha scattering at large angles and energy of the order of $\mathrm{TeV}$. Currently this value is not available. Finally it is necessary to re-run this analysis for energies of $\sqrt{s}=3 \mathrm{TeV}$ and $\sqrt{s}=10 \mathrm{TeV}$ and compare with the result obtained at $\sqrt{s}=1.5 \mathrm{TeV}$.

This work was performed within the Muon Collider Detector Design and Performance group [13]. 


\section{References}

[1] S. van der Meer, Calibration of the effective beam height in the ISR, Tech. Rep. CERN-ISR-PO-68-31 (1968).

[2] BeLLE-II collaboration, Measurement of the integrated luminosity of the Phase 2 data of the Belle II experiment, Chin. Phys. C 44 (2020) 021001 [1910.05365].

[3] BESIII collaboration, Precision measurement of the integrated luminosity of the data taken by BESIII at center of mass energies between $3.810 \mathrm{GeV}$ and $4.600 \mathrm{GeV}$, Chin. Phys. C 39 (2015) 093001 [1503.03408].

[4] C.M. Carloni Calame, H. Czyz, J. Gluza, M. Gunia, G. Montagna, O. Nicrosini et al., NNLO massive corrections to Bhabha scattering and theoretical precision of BabaYaga@NLO, Nucl. Phys. B Proc. Suppl. 225-227 (2012) 293 [1112 . 2851].

[5] K. Long, D. Lucchesi, M. Palmer, N. Pastrone, D. Schulte and V. Shiltsev, Muon colliders to expand frontiers of particle physics, Nature Phys. 17 (2021) 289 [2007. 15684].

[6] F. Collamati, C. Curatolo, D. Lucchesi, A. Mereghetti, N. Mokhov, M. Palmer et al., Advanced assessment of Beam Induced Background at a Muon Collider, 2105. 09116.

[7] Y.I. Alexahin, E. Gianfelice-Wendt, V.V. Kashikhin, N.V. Mokhov, A.V. Zlobin and V.Y. Alexakhin, Muon collider interaction region design, Phys. Rev. ST Accel. Beams 14 (2011) 061001 [1204.5739].

[8] N.V. Mokhov, S.I. Striganov and I.S. Tropin, Reducing Backgrounds in the Higgs Factory Muon Collider Detector, in 5th International Particle Accelerator Conference, pp. 1081-1083, 6, 2014, DOI [1409. 1939].

[9] M. Casarsa et al., Detector Performance Studies at a Muon Collider, PoS ICHEP2020 (2021) 826.

[10] CLICDP collaboration, A detector for CLIC: main parameters and performance, 1812.07337.

[11] T. Sjöstrand, The PYTHIA Event Generator: Past, Present and Future, Comput. Phys. Commun. 246 (2020) 106910 [1907.09874].

[12] J. Marshall and M. Thomson, Pandora particle flow algorithm, 1308.4537v1.

[13] https://muoncollider.web.cern.ch/design/muon-collider-detector. 